\title{
Dispersive Estimates for Schrödinger Operators in the Presence of a Resonance and/or an Eigenvalue at Zero Energy in Dimension Three: I
}

\author{
M. Burak Erdoğan and Wilhelm Schlag
}

Communicated by Charles Li, received October 25, 2004.

\begin{abstract}
We prove dispersive estimates for Schrödinger equations in three dimensions without making any assumptions on zero energy.
\end{abstract}

\section{Contents}

1. Introduction

2. Scalar case 360

$\begin{array}{ll}\text { References } & 379\end{array}$

\section{Introduction}

Consider the Schrödinger operator $H=-\Delta+V$ in $\mathbb{R}^{3}$, where $V$ is a real-valued potential. Let $P_{a c}$ be the orthogonal projection onto the absolutely continuous subspace of $L^{2}\left(\mathbb{R}^{3}\right)$ which is determined by $H$. In [JouSofSog], [Yaj1], [RodSch], $[$ GolSch] $]$ and $[\mathbf{G o l}], L^{1}\left(\mathbb{R}^{3}\right) \rightarrow L^{\infty}\left(\mathbb{R}^{3}\right)$ dispersive estimates for the time evolution $e^{i t H} P_{a c}$ were investigated under various decay assumptions on the potential $V$ and the assumption that zero is neither an eigenvalue nor a resonance of $H$. Recall that zero energy is a resonance iff there is $f \in L^{2,-\sigma}\left(\mathbb{R}^{3}\right) \backslash L^{2}\left(\mathbb{R}^{3}\right)$ for all $\sigma>\frac{1}{2}$ so that $H f=0$. Here $L^{2,-\sigma}=\langle x\rangle^{\sigma} L^{2}$ are the usual weighted $L^{2}$ spaces and $\langle x\rangle:=\left(1+|x|^{2}\right)^{\frac{1}{2}}$.

1991 Mathematics Subject Classification. Primary 35Q40; Secondary .

Key words and phrases. Dispersive estimates, resonances.

This work was done in June of 2004, while the first author visited Caltech and he wishes to thank that institution for its hospitality and support. The first author was partially supported by the NSF grant DMS-0303413. The second author was partially supported by a Sloan fellowship and the NSF grant DMS-0300081. 
In the first part of this paper we investigate dispersive estimates when there is a resonance at energy zero. It is well-known, see Rauch [Rau], Jensen and Kato [JenKat], and Murata [Mur], that the decay in that case is $t^{-\frac{1}{2}}$. Moreover, these authors derived expansions of the evolution into inverse powers of time in weighted $L^{2}\left(\mathbb{R}^{3}\right)$ spaces. Here, we obtain such expansions with respect to the $L^{1} \rightarrow L^{\infty}$ norm, albeit only in terms of the powers $t^{-\frac{1}{2}}$ and $t^{-\frac{3}{2}}$. Our results will require decay of the form

$$
|V(x)| \leq C\langle x\rangle^{-\beta} \text {, }
$$

for some $\beta>0$. Our goal was brevity rather than optimality. In particular, it was not our intention to obtain the minimal value of $\beta$, and our results can surely be improved in that regard. Our first result is for the case when zero is only a resonance, but not an eigenvalue.

TheOREM 1. Assume that $V$ satisfies (1) with $\beta>10$ and assume that there is a resonance at energy zero but that zero is not an eigenvalue. Then there is a time dependent rank one operator $F_{t}$ (see (28) below) such that

$$
\left\|e^{i t H} P_{a c}-t^{-1 / 2} F_{t}\right\|_{1 \rightarrow \infty} \leq C t^{-3 / 2}
$$

for all $t>0$ and $F_{t}$ satisfies

$$
\sup _{t}\left\|F_{t}\right\|_{L^{1} \rightarrow L^{\infty}}<\infty, \quad \limsup _{t \rightarrow \infty}\left\|F_{t}\right\|_{L^{1} \rightarrow L^{\infty}}>0 .
$$

The following case allows for any combination of resonances and/or eigenvalue at energy zero. It is important to note that the $t^{-\frac{3}{2}}$ bound is destroyed by an eigenvalue at zero, even if zero is not a resonance and even after projecting the zero eigenfunction away (this was discovered by Jensen and Kato [JenKat]).

Theorem 2. Assume that $V$ satisfies (1) with $\beta>10$ and assume that there is a resonance at energy zero and/or that zero is an eigenvalue. Then there is a time dependent operator $F_{t}$ such that

$$
\sup _{t}\left\|F_{t}\right\|_{L^{1} \rightarrow L^{\infty}}<\infty, \quad\left\|e^{i t H} P_{a c}-t^{-1 / 2} F_{t}\right\|_{1 \rightarrow \infty} \leq C t^{-3 / 2} .
$$

In all cases, the operators $F_{t}$ can be given explicitly, and they can of course be extracted from our proofs. The methods of this paper also apply to matrix Schrödinger operators, as considered for example in Cuccagna $[\mathbf{C u c}]$ or $[\mathbf{S c h}]$. Details of this will be given elsewhere.

\section{Scalar case}

Let $K_{\lambda_{0}}$ be the operator with kernel

$$
K_{\lambda_{0}}(x, y)=\frac{1}{\pi i} \int_{0}^{\infty} e^{i t \lambda^{2}} \lambda \chi_{\lambda_{0}}(\lambda)\left[R_{V}^{+}\left(\lambda^{2}\right)-R_{V}^{-}\left(\lambda^{2}\right)\right](x, y) d \lambda,
$$

where

$$
R_{V}^{ \pm}\left(\lambda^{2}\right)=R_{V}\left(\lambda^{2} \pm i 0\right)=\left(H-\left(\lambda^{2} \pm i 0\right)\right)^{-1}
$$

is the perturbed resolvent. By the limiting absorption principle, these boundary values are bounded operators on weighted $L^{2}$-spaces, see e.g. [Agm]. Here $\chi$ is an even smooth function supported in $[-1,1]$ and $\chi(x)=1$ for $|x|<1 / 2 ; \chi_{\lambda_{0}}(\lambda)=$ $\chi\left(\lambda / \lambda_{0}\right)$. The high energies were studied in [GolSch]: 
Theorem 3. [GolSch] Assume that $V$ satisfies (1) with some $\beta>3$, then for any $\lambda_{0}>0$

$$
\left\|e^{i t H} P_{a c}-K_{\lambda_{0}}\right\|_{1 \rightarrow \infty} \leq C_{\lambda_{0}} t^{-3 / 2} .
$$

Hence, in the proof of Theorem 1 and Theorem 2, it suffices to consider the operator $K_{\lambda_{0}}$ for some $\lambda_{0}$. One can rewrite the kernel of $K_{\lambda_{0}}$ as

$$
K_{\lambda_{0}}(x, y)=\frac{1}{\pi i} \int_{-\infty}^{\infty} e^{i t \lambda^{2}} \lambda \chi_{\lambda_{0}}(\lambda) R_{V}\left((\lambda+i 0)^{2}\right)(x, y) d \lambda,
$$

Note that $R\left((\lambda+i 0)^{2}\right)(x, y)$ is not an even function of $\lambda$; rather, we have

$$
R_{V}\left((\lambda+i 0)^{2}\right)(x, y)=\overline{R_{V}\left((-\lambda+i 0)^{2}\right)(x, y)} .
$$

2.1. Resolvent expansions at zero energy. In this section, following [JenNen], we obtain resolvent expansions at the threshold $\lambda=0$ in the presence of a resonance. This is of course similar to Jensen and Kato [JenKat], but we prefer to work with the $L^{2}$-based approach from [JenNen]. For $j=0,1,2, \ldots$, let $G_{j}$ be the operator with the kernel

$$
G_{j}(x, y)=\frac{1}{4 \pi j !}|x-y|^{j-1} .
$$

Recall that for each $J=0,1,2, \ldots$,

$$
R_{0}\left(\lambda^{2}\right)=\sum_{j=0}^{J}(i \lambda)^{j} G_{j}+o\left(\lambda^{J}\right), \text { as } \lambda \rightarrow 0 .
$$

This expansion is valid in the space, $H S_{L^{2, \sigma} \rightarrow L^{2,-\sigma}}$, of Hilbert-Schmidt operators between $L^{2, \sigma}$ and $L^{2,-\sigma}$ for $\sigma>\max ((2 J+1) / 2,3 / 2)$.

Let $U(x)=1$ if $V(x) \geq 0$ and $U(x)=-1$ if $V(x)<0, v=|V|^{1 / 2}$ and $w=v U$. We have

$$
V=U v^{2}=w v
$$

We use the symmetric resolvent identity, valid for $\Im \lambda>0$ :

$$
R_{V}\left(\lambda^{2}\right)=R_{0}\left(\lambda^{2}\right)-R_{0}\left(\lambda^{2}\right) v A(\lambda)^{-1} v R_{0}\left(\lambda^{2}\right),
$$

where

$$
\begin{aligned}
A(\lambda) & =U+v R_{0}\left(\lambda^{2}\right) v=\left(U+v G_{0} v\right)+\lambda \frac{v\left[R_{0}\left(\lambda^{2}\right)-G_{0}\right] v}{\lambda} \\
& =: A_{0}+\lambda A_{1}(\lambda) .
\end{aligned}
$$

$A_{1}(\lambda)$ has the kernel

$$
\begin{aligned}
A_{1}(\lambda)(x, y) & =\frac{1}{\lambda} v(x) \frac{e^{i \lambda|x-y|}-1}{4 \pi|x-y|} v(y), \\
\left|A_{1}(\lambda)(x, y)\right| & \leq \frac{1}{4 \pi}|v(x)||v(y)| .
\end{aligned}
$$

Therefore, $A_{1}(\lambda) \in H S:=H S_{L^{2} \rightarrow L^{2}}$ provided $\langle x\rangle^{\frac{3}{2}+} v(x) \in L^{\infty}$. Also note that

$$
A_{1}(0)=i v G_{1} v=\frac{i \alpha}{4 \pi} P_{v}, \quad \alpha=\|V\|_{1},
$$

where $P_{v}$ is the orthogonal projection onto $\operatorname{span}(v)$. It is important to realize that $A(\lambda)$ has a natural meaning for $\lambda \in \mathbb{R}$ via the limit $\mathbb{R}+i 0$. 
First, we consider the expansions of $A(\lambda)^{-1}$ for $\lambda$ close to zero as in [JenNen]. The following lemma (Corollary 2.2 in [JenNen]) is our main tool. Note the similarity between (7) and the symmetric resolvent identity.

Lemma 4. [JenNen] Let $F \subset \mathbb{C} \backslash\{0\}$ have zero as an accumulation point. Let $A(z), z \in F$, be a family of bounded operators of the form

$$
A(z)=A_{0}+z A_{1}(z)
$$

with $A_{1}(z)$ uniformly bounded as $z \rightarrow 0$. Suppose that 0 is an isolated point of the spectrum of $A_{0}$, and let $S$ be the corresponding Riesz projection. Assume that $\operatorname{rank}(S)<\infty$. Then for sufficiently small $z \in F$ the operators

$$
B(z):=\frac{1}{z}\left(S-S(A(z)+S)^{-1} S\right)
$$

are well-defined and bounded on $\mathcal{H}$. Moreover, if $A_{0}=A_{0}^{*}$, then they are uniformly bounded as $z \rightarrow 0$. The operator $A(z)$ has a bounded inverse in $\mathcal{H}$ if and only if $B(z)$ has a bounded inverse in $S \mathcal{H}$, and in this case

$$
A(z)^{-1}=(A(z)+S)^{-1}+\frac{1}{z}(A(z)+S)^{-1} S B(z)^{-1} S(A(z)+S)^{-1} .
$$

Proof. It is a standard fact that

$$
\operatorname{Ran}(S) \supset \bigcup_{n=1}^{\infty} \operatorname{ker}\left(A_{0}^{n}\right) .
$$

By our assumption $\operatorname{rank}(S)<\infty$ we have equality here, and $\left(A_{0}+S\right)^{-1}$ has a bounded inverse. Hence, $A(z)+S$ also has a bounded inverse for small $z$, as can be seen from the usual Neuman series. Therefore, $B(z)$ is well-defined for small $z$ and bounded. Moreover, if $A_{0}$ is self-adjoint, then

$$
S-S\left(A_{0}+S\right)^{-1} S=0
$$

which implies that $B(z)=O(1)$ as $z \rightarrow 0$. Suppose $B(z)$ is invertible on $S \mathcal{H}$. Denote the right-hand side of (7) by $T(z)$. Then

$$
\begin{aligned}
T(z) A(z)= & A(z) T(z) \\
= & I+\frac{1}{z} S B(z)^{-1} S(A(z)+S)^{-1}-S(A(z)+S)^{-1} \\
& -\frac{1}{z} S(A(z)+S)^{-1} S B(z)^{-1} S(A(z)+S)^{-1} \\
= & I+\frac{1}{z} S B(z)^{-1} S(A(z)+S)^{-1}-S(A(z)+S)^{-1} \\
& -\frac{1}{z}(S-z B(z)) B(z)^{-1} S(A(z)+S)^{-1}=I .
\end{aligned}
$$

Conversely, suppose that $A(z)$ is invertible. Define

$$
D(z):=z\left(S+S A(z)^{-1} S\right)=z(A(z)+S)\left[A(z)^{-1}-(A(z)+S)^{-1}\right](A(z)+S) .
$$

Then

$$
\begin{aligned}
B(z) D(z) & =D(z) B(z) \\
& =S+S A(z)^{-1} S-S(A(z)+S)^{-1} S-S(A(z)+S)^{-1} S A(z)^{-1} S \\
& =S+S(A(z)+S)^{-1} S A(z)^{-1} S-S(A(z)+S)^{-1} S A(z)^{-1} S=S,
\end{aligned}
$$

so that $D(z)$ is the inverse of $B(z)$ on $S \mathcal{H}$. 
Note that $A_{0}$ as in (6) is a compact perturbation of $U$ and that the essential spectrum of $U$ is contained in $\{-1,1\}$. Moreover, $A_{0}$ is self adjoint. Therefore, 0 is an isolated point of the spectrum of $A_{0}$ and $\operatorname{dim}\left(\operatorname{ker}_{A_{0}}\right)<\infty$. Let $S_{1}$ be the corresponding Riesz projection. Since $A_{0}$ is self adjoint, $S_{1}$ is the orthogonal projection onto the kernel of $A_{0}$ and we have

$$
S_{1}=\left(A_{0}+S_{1}\right)^{-1} S_{1}=S_{1}\left(A_{0}+S_{1}\right)^{-1} .
$$

REMARK 1. By the resolvent identity we have

$$
\left(A_{0}+S_{1}\right)^{-1}=U-\left(A_{0}+S_{1}\right)^{-1}\left(v G_{0} v+S_{1}\right) U .
$$

Since $|V(x)| \lesssim\langle x\rangle^{-3-}$ and $S_{1}$ is a finite rank operator, we have $\left(v G_{0} v+S_{1}\right) U \in H S$, and hence $\left(A_{0}+S_{1}\right)^{-1}$ is the sum of $U$ and a Hilbert-Schmidt operator. Therefore, the operator with kernel $\left|\left(A_{0}+S_{1}\right)^{-1}(x, y)\right|$ is bounded in $L^{2}$. This remark will be useful below when we consider dispersive estimates.

We choose $\lambda_{0}>0$ sufficiently small so that $A(\lambda)+S_{1}$ is invertible for $|\lambda|<\lambda_{0}$. Using Lemma 4, we see that, for $|\lambda|<\lambda_{0}, A(\lambda)$ is invertible if and only if

$$
m(\lambda)=\frac{S_{1}-S_{1}\left(A(\lambda)+S_{1}\right)^{-1} S_{1}}{\lambda}
$$

is invertible on $S_{1} L^{2}$ and in this case

(9) $A(\lambda)^{-1}=\left(A(\lambda)+S_{1}\right)^{-1}+\frac{1}{\lambda}\left(A(\lambda)+S_{1}\right)^{-1} S_{1} m(\lambda)^{-1} S_{1}\left(A(\lambda)+S_{1}\right)^{-1}$.

If $\lambda_{0}$ is sufficiently small, then

$$
\left(A(\lambda)+S_{1}\right)^{-1}=\left(A_{0}+S_{1}\right)^{-1}+\sum_{k=1}^{\infty}(-1)^{k} \lambda^{k}\left(A_{0}+S_{1}\right)^{-1}\left(A_{1}(\lambda)\left(A_{0}+S_{1}\right)^{-1}\right)^{k} .
$$

Plugging this into the definition of $m(\lambda)$ and using (8), we obtain

$$
\begin{aligned}
m(\lambda) & =S_{1} A_{1}(\lambda) S_{1}+\sum_{k=1}^{\infty}(-1)^{k} \lambda^{k} S_{1}\left(A_{1}(\lambda)\left(A_{0}+S_{1}\right)^{-1}\right)^{k+1} S_{1} \\
& =m(0)+\lambda m_{1}(\lambda),
\end{aligned}
$$

where

$$
\begin{aligned}
m(0) & =S_{1} A_{1}(0) S_{1}=\frac{i \alpha}{4 \pi} S_{1} P_{v} S_{1} \\
m_{1}(\lambda) & =S_{1} \frac{A_{1}(\lambda)-A_{1}(0)}{\lambda} S_{1}+\sum_{k=1}^{\infty}(-1)^{k} \lambda^{k} S_{1}\left(A_{1}(\lambda)\left(A_{0}+S_{1}\right)^{-1}\right)^{k+1} S_{1} .
\end{aligned}
$$

If $m(0)$ is invertible in $S_{1} L^{2}$, then we can invert $m(\lambda)$ for small $\lambda$ using Neuman series and hence obtain an expansion for $A(\lambda)^{-1}$. Since $m(0)$ has rank one, this can only occur if $\operatorname{rank}\left(S_{1}\right)=1$.

Otherwise, let $S_{2}: S_{1} L^{2} \rightarrow S_{1} L^{2}$ be the orthogonal projection onto the kernel of $m(0)$ where the latter operates on $S_{1} L^{2}$. As above, $m(\lambda)+S_{2}$ is invertible in $S_{1} L^{2}$ for $|\lambda|<\lambda_{0}$ (we choose a smaller $\lambda_{0}$ if necessary), and

$$
S_{2}=S_{2}\left(m(0)+S_{2}\right)^{-1}=\left(m(0)+S_{2}\right)^{-1} S_{2} .
$$


Lemma 4 asserts that $m(\lambda)$ is invertible on $S_{1} L^{2}$ if and only if

$$
b(\lambda)=\frac{S_{2}-S_{2}\left(m(\lambda)+S_{2}\right)^{-1} S_{2}}{\lambda}
$$

is invertible on $S_{2} L^{2}$ and

$(12)_{h}(\lambda)^{-1}=\left(m(\lambda)+S_{2}\right)^{-1}+\frac{1}{\lambda}\left(m(\lambda)+S_{2}\right)^{-1} S_{2} b(\lambda)^{-1} S_{2}\left(m(\lambda)+S_{2}\right)^{-1}$.

Note that

$$
\left(m(\lambda)+S_{2}\right)^{-1}=\left(m(0)+S_{2}\right)^{-1}+\sum_{k=1}^{\infty}(-1)^{k} \lambda^{k}\left(m(0)+S_{2}\right)^{-1}\left(m_{1}(\lambda)\left(m(0)+S_{2}\right)^{-1}\right)^{k} .
$$

Plugging this into the definition of $b(\lambda)$ and using (11), we obtain

$$
\begin{aligned}
b(\lambda) & =S_{2} m_{1}(\lambda) S_{2}+\sum_{k=1}^{\infty}(-1)^{k} \lambda^{k} S_{2}\left(m_{1}(\lambda)\left(m(0)+S_{2}\right)^{-1}\right)^{k+1} S_{2} \\
& =: b(0)+\lambda b_{1}(\lambda),
\end{aligned}
$$

where $b(0)=S_{2} m_{1}(0) S_{2}$ and

$$
b_{1}(\lambda)=\frac{S_{2}\left[m_{1}(\lambda)-m_{1}(0)\right] S_{2}}{\lambda}+\frac{1}{\lambda} \sum_{k=1}^{\infty}(-1)^{k} \lambda^{k} S_{2}\left(m_{1}(\lambda)\left(m(0)+S_{2}\right)^{-1}\right)^{k+1} S_{2} .
$$

A simple calculation using (4) (with $J=2$ ) and $S_{2} S_{1}=S_{1} S_{2}=S_{2}$ shows that

$$
b(0)=-S_{2} v G_{2} v S_{2} .
$$

Since $G_{2} \in H S_{L^{2, \sigma} \rightarrow L^{2,-\sigma}}$ for $\sigma>5 / 2$, we have $b(0) \in H S$ if $|V(x)| \lesssim\langle x\rangle^{-5-\varepsilon}$.

Below, we characterize the spaces $S_{1} L^{2}, S_{2} L^{2}$ and also prove that $b(0)$ is always invertible on $S_{2} L^{2}$. Therefore, for small $\lambda, b(\lambda)$ is invertible. This proves that $A(\lambda)$ is invertible for $0<|\lambda|<\lambda_{0}$. Using (9) and (12), we obtain

$$
\begin{aligned}
A(\lambda)^{-1} & =\Gamma_{1}(\lambda) \\
& +\frac{1}{\lambda} \Gamma_{1}(\lambda) S_{1} \Gamma_{2}(\lambda) S_{1} \Gamma_{1}(\lambda) \\
& +\frac{1}{\lambda^{2}} \Gamma_{1}(\lambda) S_{1} \Gamma_{2}(\lambda) S_{2} b(\lambda)^{-1} S_{2} \Gamma_{2}(\lambda) S_{1} \Gamma_{1}(\lambda),
\end{aligned}
$$

where

$$
\Gamma_{1}(\lambda)=\left(A(\lambda)+S_{1}\right)^{-1}, \text { and } \Gamma_{2}(\lambda)=\left(m(\lambda)+S_{2}\right)^{-1} .
$$

Note that this formula is also valid in the case $S_{2}=0$.

Lemma 5. Assume $|V(x)| \lesssim\langle x\rangle^{-3-\varepsilon}$. Then $f \in S_{1} L^{2} \backslash\{0\}$ if and only if $f=w g$ for some $g \in L^{2,-\frac{1}{2}-} \backslash\{0\}$ such that

$$
-\Delta g+V g=0 \text { in } \mathcal{S}^{\prime} .
$$

Proof. First recall that, for $g \in L^{2,-\frac{1}{2}-}$, (16) holds if and only if

$$
\left(I+G_{0} V\right) g=0,
$$


see Lemma 2.4 in [JenKat]. Suppose $f \in S_{1} L^{2} \backslash\{0\}$. Then

$$
\begin{gathered}
\left(A_{0} f\right)(x)=U(x) f(x)+\frac{v(x)}{4 \pi} \int \frac{v(y) f(y)}{|x-y|} d y=0 \\
\Rightarrow f(x)+\frac{w(x)}{4 \pi} \int \frac{v(y) f(y)}{|x-y|} d y=0 .
\end{gathered}
$$

Let

$$
g(x)=-\frac{1}{4 \pi} \int_{\mathbb{R}^{3}} \frac{v(y) f(y)}{|x-y|} d y .
$$

Note that $g \in L^{2,-\frac{1}{2}-}$ and $f(x)=w(x) g(x)$ for each $x$. Moreover, (16) holds since

$$
g(x)=-\frac{1}{4 \pi} \int_{\mathbb{R}^{3}} \frac{v(y) f(y)}{|x-y|} d y=-\frac{1}{4 \pi} \int_{\mathbb{R}^{3}} \frac{V(y) g(y)}{|x-y|} d y=-\left[G_{0} V g\right](x) .
$$

Conversely, assume $f=w g$ for some $g$ as in the hypothesis. Then $f \in L^{2,1+}$ and

$$
\begin{aligned}
A_{0} f(x) & =U(x) f(x)+\frac{v(x)}{4 \pi} \int \frac{v(y) f(y)}{|x-y|} d y \\
& =v(x) g(x)+\frac{v(x)}{4 \pi} \int \frac{V(y) g(y)}{|x-y|} d y=v\left(I+G_{0} V\right) g=0 .
\end{aligned}
$$

Note that since $g$ is not identically zero, $V g \neq 0$, and hence $f \neq 0$.

By Lemma 5 , we see that $f \in S_{1} L^{2}$ implies $f \in L^{2,1+}$.

Lemma 6. Assume $|V(x)| \lesssim\langle x\rangle^{-3-\varepsilon}$. Then $f \in S_{2} L^{2} \backslash\{0\}$ if and only if $f=w g$ for some $g \in L^{2} \backslash\{0\}$ such that

$$
-\Delta g+V g=0 \text { in } \mathcal{S}^{\prime}
$$

Proof. Suppose $f \in S_{2} L^{2} \backslash\{0\}$. Note that $S_{2} L^{2} \subset S_{1} L^{2}$ and, by Lemma 5 , we have $f=w g$ for some $g \in L^{2,-\frac{1}{2}-} \backslash\{0\}$ such that $-\Delta g+V g=0$ in $\mathcal{S}^{\prime}$. By the definition of $S_{2}$, we have

$$
S_{1} P_{v} f=0 .
$$

Note that $S_{1} P_{v} f=0$ if and only if

$$
S_{1} v=0 \text { or } P_{v} f=0 .
$$

In the first case, $S_{2}=S_{1}$ and $P_{v} f=0$ for any $f \in S_{2} L^{2}$. We have the same conclusion in the second case. Thus,

$$
\int_{\mathbb{R}^{3}} v(y) f(y) d y=0 .
$$

Using this and (18), we obtain

$$
g(x)=-\frac{1}{4 \pi} \int_{\mathbb{R}^{3}}\left[\frac{1}{|x-y|}-\frac{1}{1+|x|}\right] v(y) f(y) d y \in L^{2, \frac{1}{2}-} .
$$

This is because

$$
\left|\frac{1}{|x-y|}-\frac{1}{1+|x|}\right| \leq \frac{1+|y|}{|x-y|(1+|x|)}
$$

and $f \in L^{2,1+}$. 
Conversely, assume $f=w g$ for some $g$ as in the hypothesis. Then

$$
g(x)=-\frac{1}{4 \pi} \int_{\mathbb{R}^{3}}\left[\frac{1}{|x-y|}-\frac{1}{1+|x|}\right] v(y) f(y) d y-\frac{1}{4 \pi(1+|x|)} \int_{\mathbb{R}^{3}} v(y) f(y) d y .
$$

By (19) the first summand is in $L^{2}$. Therefore

$$
\left[\int_{\mathbb{R}^{3}} v(y) f(y) d y\right] \frac{1}{1+|\cdot|} \in L^{2}\left(\mathbb{R}^{3}\right) .
$$

Thus, $\int v(y) f(y) d y=0$ and $f \in S_{2} L^{2} \backslash\{0\}$.

Lemma 7. Assume $|V(x)| \lesssim\langle x\rangle^{-5-\varepsilon}$. Then, as an operator in $S_{2} L^{2}$, the kernel of $b(0)$ is trivial.

Proof. Assume that for some $f \in S_{2} L^{2}, b(0) f=0$, i.e.,

$$
\left\langle G_{2} v f, v f\right\rangle=0 \text {. }
$$

From the proof of Lemma (6), we have

$$
\int_{\mathbb{R}^{3}} f(y) v(y) d y=0 .
$$

Using this and (4) (with $J=2$ ), we obtain

$$
\begin{aligned}
0 & =\left\langle G_{2} v f, v f\right\rangle \\
& =\lim _{\lambda \rightarrow 0}\left\langle\frac{R_{0}\left(\lambda^{2}\right)-G_{0}}{\lambda^{2}} v f, v f\right\rangle \\
& =\lim _{\lambda \rightarrow 0} \frac{1}{\lambda^{2}} \int\left(\left(\xi^{2}+\lambda^{2}\right)^{-1}-\xi^{-2}\right) \widehat{v f}(\xi) \widehat{\widehat{v f}}(\xi) d \xi \\
& =\lim _{\lambda \rightarrow 0} \int \frac{1}{\xi^{2}\left(\xi^{2}+\lambda^{2}\right)}|\widehat{v f}(\xi)|^{2} d \xi \\
& \left.=\int \frac{|\widehat{v f}(\xi)|^{2}}{\xi^{4}} d \xi \quad \text { by the Monot. Conv. Thm. }\right) \\
& =\left\langle R_{0}(0) v f, R_{0}(0) v f\right\rangle \quad \Rightarrow \widehat{v f}=0 \Rightarrow v f=0 .
\end{aligned}
$$

Using this in (17), we obtain $f=0$.

2.2. Dispersive estimate when zero is not an eigenvalue. In this section, we prove Theorem 1 . When zero is not an eigenvalue, $S_{2}=0$ and (15) reduces to $(20) A(\lambda)^{-1}=\left(A(\lambda)+S_{1}\right)^{-1}+\frac{1}{\lambda}\left(A(\lambda)+S_{1}\right)^{-1} S_{1} m(\lambda)^{-1} S_{1}\left(A(\lambda)+S_{1}\right)^{-1}$, where

$$
\begin{aligned}
\left(A(\lambda)+S_{1}\right)^{-1} & =\left(A_{0}+S_{1}\right)^{-1}+\sum_{k=1}^{\infty}(-1)^{k} \lambda^{k}\left(A_{0}+S_{1}\right)^{-1}\left[A_{1}(\lambda)\left(A_{0}+S_{1}\right)^{-1}\right]^{k} \\
& =:\left(A_{0}+S_{1}\right)^{-1}+\lambda E_{1}(\lambda), \\
m(\lambda)^{-1} & =m(0)^{-1}+\sum_{k=1}^{\infty}(-1)^{k} \lambda^{k} m(0)^{-1}\left[m_{1}(\lambda) m(0)^{-1}\right]^{k} \\
& =: m(0)^{-1}+\lambda E_{2}(\lambda) .
\end{aligned}
$$


Thus, using (8), we obtain

$$
\begin{aligned}
A(\lambda)^{-1} & =\frac{1}{\lambda} S_{1} m(0)^{-1} S_{1} \\
& +\left(A(\lambda)+S_{1}\right)^{-1} \\
& +E_{1}(\lambda) S_{1} m(\lambda)^{-1} S_{1}\left(A(\lambda)+S_{1}\right)^{-1} \\
& +\left(A(\lambda)+S_{1}\right)^{-1} S_{1} E_{2}(\lambda) S_{1}\left(A(\lambda)+S_{1}\right)^{-1} \\
& +\left(A(\lambda)+S_{1}\right)^{-1} S_{1} m(\lambda)^{-1} S_{1} E_{1}(\lambda) \\
& =: \frac{1}{\lambda} S+E(\lambda) .
\end{aligned}
$$

Note that $S$ is a rank one operator. Plugging (22) into (5), we have

$$
\begin{aligned}
R_{V}\left(\lambda^{2}\right) & =-\frac{1}{\lambda} R_{0}\left(\lambda^{2}\right) v S v R_{0}\left(\lambda^{2}\right) \\
& +R_{0}\left(\lambda^{2}\right)-R_{0}\left(\lambda^{2}\right) v E(\lambda) v R_{0}\left(\lambda^{2}\right)
\end{aligned}
$$

Using this in (3), we get

$$
K_{\lambda_{0}}(x, y)=K_{1}(x, y)+K_{2}(x, y)-K_{3}(x, y)
$$

where

$$
\begin{aligned}
& K_{1}(x, y)=\frac{-i}{16 \pi^{3}} \int_{-\infty}^{\infty} \int_{\mathbb{R}^{6}} e^{i t \lambda^{2}} \chi_{\lambda_{0}}(\lambda) \frac{e^{i \lambda\left(\left|x-u_{1}\right|+\left|y-u_{2}\right|\right)}}{\left|x-u_{1}\right|\left|y-u_{2}\right|} v\left(u_{1}\right) S\left(u_{1}, u_{2}\right) v\left(u_{2}\right) d u_{1} d u_{2} d \lambda \\
& K_{2}(x, y)=\int_{-\infty}^{\infty} e^{i t \lambda^{2}} \lambda \chi_{\lambda_{0}}(\lambda) R_{0}\left(\lambda^{2}\right)(x, y) d \lambda \\
& (23) \\
& K_{3}(x, y)=\int_{-\infty}^{\infty} e^{i t \lambda^{2}} \lambda \chi_{\lambda_{0}}(\lambda)\left[R_{0}\left(\lambda^{2}\right) v E(\lambda) v R_{0}\left(\lambda^{2}\right)\right](x, y) d \lambda .
\end{aligned}
$$

First, we deal with $K_{1}$. Note that

$$
\begin{aligned}
& K_{1}(x, y)= \\
& \frac{-i}{16 \pi^{3}} \int_{\mathbb{R}^{6}} \int_{-\infty}^{\infty} e^{i t \lambda^{2}} \chi_{\lambda_{0}}(\lambda) \frac{\cos \left(\lambda\left(\left|x-u_{1}\right|+\left|y-u_{2}\right|\right)\right)}{\left|x-u_{1}\right|\left|y-u_{2}\right|} v\left(u_{1}\right) S\left(u_{1}, u_{2}\right) v\left(u_{2}\right) d u_{1} d u_{2} d \lambda .
\end{aligned}
$$


We have

$$
\begin{aligned}
\int_{-\infty}^{\infty} t^{1 / 2} e^{i t \lambda^{2}} \chi_{\lambda_{0}}(\lambda) \cos (\lambda a) d \lambda & =\int_{-\infty}^{\infty}\left(t^{1 / 2} e^{i t(\cdot)^{2}}\right)^{\vee}(u)\left(\chi_{\lambda_{0}}(\cdot) \cos (\cdot a)\right)^{\wedge}(u) d u \\
& =c \int_{-\infty}^{\infty} e^{i u^{2} / 4 t}\left(\widehat{\chi_{\lambda_{0}}}(u+a)+\widehat{\chi_{\lambda_{0}}}(u-a)\right) d u \\
& =c \int_{-\infty}^{\infty} e^{i\left(u^{2}+a^{2}\right) / 4 t} \cos \left(\frac{u a}{2 t}\right) \widehat{\chi_{\lambda_{0}}}(u) d u \\
& =c \int_{-\infty}^{\infty} e^{i\left(u^{2}+a^{2}\right) / 4 t} \widehat{\chi_{\lambda_{0}}}(u) d u \\
& +c \int_{-\infty}^{\infty} e^{i\left(u^{2}+a^{2}\right) / 4 t}\left(\cos \left(\frac{u a}{2 t}\right)-1\right) \widehat{\chi_{\lambda_{0}}}(u) d u \\
& =: C_{1}(t, a)+C_{2}(t, a) .
\end{aligned}
$$

Using this in (24), we obtain

$$
\begin{aligned}
K_{1}(x, y) & =\frac{-i t^{-1 / 2}}{16 \pi^{3}} \int_{\mathbb{R}^{6}} \frac{C_{1}\left(t,\left|x-u_{1}\right|+\left|y-u_{2}\right|\right)}{\left|x-u_{1}\right|\left|y-u_{2}\right|} v\left(u_{1}\right) S\left(u_{1}, u_{2}\right) v\left(u_{2}\right) d u_{1} d u_{2} \\
& +\frac{-i t^{-1 / 2}}{16 \pi^{3}} \int_{\mathbb{R}^{6}} \frac{C_{2}\left(t,\left|x-u_{1}\right|+\left|y-u_{2}\right|\right)}{\left|x-u_{1}\right|\left|y-u_{2}\right|} v\left(u_{1}\right) S\left(u_{1}, u_{2}\right) v\left(u_{2}\right) d u_{1} d u_{2} \\
& =: K_{11}(x, y)+K_{12}(x, y) .
\end{aligned}
$$

Note that

$$
\left|C_{2}(t, a)\right| \leq c \frac{|a|}{t}
$$

Thus,

$$
\begin{aligned}
\left|K_{12}(x, y)\right| & \leq c t^{-3 / 2} \int_{\mathbb{R}^{6}}\left(\frac{1}{\left|x-u_{1}\right|}+\frac{1}{\left|y-u_{2}\right|}\right)\left|v\left(u_{1}\right) \| v\left(u_{2}\right)\right|\left|S\left(u_{1}, u_{2}\right)\right| d u_{1} d u_{2} \\
& \lesssim t^{-3 / 2}\left[\left\|\frac{v(\cdot)}{|x-\cdot|}\right\|_{2}+\left\|\frac{v(\cdot)}{|y-\cdot|}\right\|_{2}\right]\||S|\|_{2 \rightarrow 2}\|v\|_{2} \\
& \lesssim t^{-3 / 2} .
\end{aligned}
$$

The last inequality follows from the fact that $S$ is a rank one operator and the following calculation which holds for $v \in L^{2} \cap L^{\infty}$;

$$
\begin{aligned}
\left\|\frac{|v(\cdot)|}{|x-\cdot|}\right\|_{2}^{2} & =\int_{|x-u|<1} \frac{|v(u)|^{2}}{|x-u|^{2}} d u+\int_{|x-u|>1} \frac{|v(u)|^{2}}{|x-u|^{2}} d u \\
& \lesssim \int_{|u|<1} \frac{1}{|u|^{2}} d u+\int_{\mathbb{R}^{3}}|v(u)|^{2} d u \lesssim 1
\end{aligned}
$$

Now, we consider $K_{11}$. Note that

$$
C_{1}(t, a)=e^{i a^{2} / 4 t} h(t)
$$


where $h(t)$ is a smooth function which converges to $c$ as $t$ tends to $\infty$. We have

$$
\begin{aligned}
K_{11}(x, y) & =\frac{-i h(t)}{16 \pi^{3} t^{1 / 2}} \int_{\mathbb{R}^{6}} \frac{e^{i\left|x-u_{1}\right|^{2} / 4 t} e^{i\left|y-u_{2}\right|^{2} / 4 t}}{\left|x-u_{1}\right|\left|y-u_{2}\right|} v\left(u_{1}\right) S\left(u_{1}, u_{2}\right) v\left(u_{2}\right) d u_{1} d u_{2} \\
& -\frac{i h(t)}{16 \pi^{3} t^{1 / 2}} \int_{\mathbb{R}^{6}} \frac{e^{\frac{i\left(\left|x-u_{1}\right|+\left|y-u_{2}\right|\right)^{2}}{4 t}}-e^{\frac{i\left(\left|x-u_{1}\right|^{2}+\left|y-u_{2}\right|^{2}\right)}{4 t}}}{\left|x-u_{1}\right|\left|y-u_{2}\right|} v\left(u_{1}\right) S\left(u_{1}, u_{2}\right) v\left(u_{2}\right) d u_{1} d u_{2} \\
& =: t^{-1 / 2} F_{t}(x, y)+K_{112}(x, y) .
\end{aligned}
$$

Since $S$ is a rank one operator, for each $t, F_{t}$ is a rank one operator. Also note that by a calculation similar to (26), we obtain $\sup _{t, x, y}\left|F_{t}(x, y)\right| \lesssim 1$. Finally, $F_{t} \neq 0$ for all $t$, and $\lim _{t \rightarrow \infty} F_{t}$ exists in the weak sense and does not vanish:

$$
\lim _{t \rightarrow \infty}\left\langle F_{t} f, g\right\rangle=\frac{-i c}{16 \pi^{3}} \int_{\mathbb{R}^{12}} \frac{f(x) \bar{g}(y)}{\left|x-u_{1}\right|\left|y-u_{2}\right|} v\left(u_{1}\right) S\left(u_{1}, u_{2}\right) v\left(u_{2}\right) d u_{1} d u_{2} d x d y
$$

for any $f, g \in \mathcal{S}$. By a similar calculation, the term $K_{112}$ is dispersive since

$$
\left|e^{i\left(\left|x-u_{1}\right|+\left|y-u_{2}\right|\right)^{2} / 4 t}-e^{i\left(\left|x-u_{1}\right|^{2}+\left|y-u_{2}\right|^{2}\right) / 4 t}\right| \lesssim \frac{\left|x-u_{1}\right|\left|y-u_{2}\right|}{t} .
$$

$K_{2}$ is the low energy part of the free evolution and hence it is dispersive. The rest of this section is devoted to the proof of

$$
\sup _{x, y}\left|K_{3}(x, y)\right| \lesssim t^{-3 / 2} .
$$

Denote

$$
\frac{d}{d \lambda}\left(\chi_{\lambda_{0}}(\lambda) R_{0}\left(\lambda^{2}\right) v E(\lambda) v R_{0}\left(\lambda^{2}\right)\right)
$$

by $\mathcal{F}_{x, y}(\lambda)$. By integration by parts we obtain

$$
K_{3}(x, y)=\frac{1}{2 i t} \int_{-\infty}^{\infty} e^{i t \lambda^{2}} \mathcal{F}_{x, y}(\lambda) d \lambda .
$$

Using Parseval's formula, we obtain

$$
K_{3}(x, y)=\frac{c}{t^{3 / 2}} \int_{-\infty}^{\infty} e^{i \xi^{2} / 4 t} \widehat{\mathcal{F}_{x, y}}(\xi) d \xi
$$

Thus, it suffices to prove that

$$
\sup _{x, y}\left\|\widehat{\mathcal{F}_{x, y}}\right\|_{L^{1}}<\infty .
$$

Recall that

$$
\mathcal{F}_{x, y}(\lambda)=\int_{\mathbb{R}^{6}} \frac{d}{d \lambda}\left[\chi_{\lambda_{0}}(\lambda) E(\lambda)\left(u_{1}, u_{2}\right) v\left(u_{1}\right) v\left(u_{2}\right) \frac{e^{i \lambda\left(\left|x-u_{1}\right|+\left|y-u_{2}\right|\right)}}{\left|x-u_{1}\right|\left|y-u_{2}\right|}\right] d u_{1} d u_{2} .
$$

Let us concentrate on the term where the derivative hits $\chi_{\lambda_{0}}(\lambda) E(\lambda)$ (the term where the derivative hits the exponential is similar):

$$
\tilde{\mathcal{F}}_{x, y}(\lambda)=\int_{\mathbb{R}^{6}}\left[\chi_{\lambda_{0}}(\lambda) E(\lambda)\right]^{\prime}\left(u_{1}, u_{2}\right) v\left(u_{1}\right) v\left(u_{2}\right) \frac{e^{i \lambda\left(\left|x-u_{1}\right|+\left|y-u_{2}\right|\right)}}{\left|x-u_{1}\right|\left|y-u_{2}\right|} d u_{1} d u_{2} .
$$


Note that

$$
\begin{aligned}
\left\|\widehat{\tilde{\mathcal{F}}_{x, y}}(\xi)\right\|_{L^{1}} & =\int_{-\infty}^{\infty} \mid \int_{\mathbb{R}^{6}}\left(\widehat{\chi_{\lambda_{0}} E}\right)^{\prime}\left(\xi+\left|x-u_{1}\right|+\left|y-u_{2}\right|\right)\left(u_{1}, u_{2}\right) \\
& \frac{v\left(u_{1}\right) v\left(u_{2}\right)}{\left|x-u_{1}\right|\left|y-u_{2}\right|} d u_{1} d u_{2} \mid d \xi \\
& \leq \int_{\mathbb{R}^{6}} \int_{-\infty}^{\infty}\left|\widehat{\left(\widehat{\chi_{\lambda_{0}} E}\right)^{\prime}}\left(\xi+\left|x-u_{1}\right|+\left|y-u_{2}\right|\right)\left(u_{1}, u_{2}\right)\right| \\
& \frac{\left|v\left(u_{1}\right)\right|\left|v\left(u_{2}\right)\right|}{\left|x-u_{1}\right|\left|y-u_{2}\right|} d \xi d u_{1} d u_{2} \\
& =\int_{\mathbb{R}^{6}} \int_{-\infty}^{\infty}\left|\widehat{\left(\chi_{\lambda_{0}} E\right)^{\prime}}(\xi)\left(u_{1}, u_{2}\right)\right| \frac{\left|v\left(u_{1}\right)\right|\left|v\left(u_{2}\right)\right|}{\left|x-u_{1}\right|\left|y-u_{2}\right|} d \xi d u_{1} d u_{2} \\
& \leq\left\|\frac{|v(\cdot)|}{|x-\cdot|}||\left|\frac{|v(\cdot)|}{|y-\cdot|}\right|_{2} \int_{-\infty}^{\infty}\right\|\left|\widehat{\left(\chi_{\lambda_{0}} E\right)^{\prime}}(\xi)\right| \|_{L^{2} \rightarrow L^{2}} d \xi \\
& \lesssim \int_{-\infty}^{\infty}\left\|\left|\widehat{\left(\widehat{\chi_{\lambda_{0}} E}\right)^{\prime}}(\xi)\right|\right\|_{L^{2} \rightarrow L^{2}} d \xi .
\end{aligned}
$$

The second line follows from Minkowski's inequality and Fubini's theorem, the third line follows from a change of variable, and the last line follows from the calculation (27). Therefore, for $\tilde{\mathcal{F}}_{x, y},(31)$ follows from

$$
\int_{-\infty}^{\infty}\left\|\left|\widehat{\left(\chi_{\lambda_{0}} E\right)^{\prime}}(\xi)\right|\right\|_{L^{2} \rightarrow L^{2}} d \xi<\infty .
$$

We shall use the following elementary lemma.

Lemma 8. For each $\lambda \in \mathbb{R}$, let $F_{1}(\lambda)$ and $F_{2}(\lambda)$ be bounded operators from $L^{2}\left(\mathbb{R}^{3}\right)$ to $L^{2}\left(\mathbb{R}^{3}\right)$ with kernels $K_{1}(\lambda)$ and $K_{2}(\lambda)$. Suppose that $K_{1}, K_{2}$ both have compact support in $\lambda$ and that $K_{j}(\cdot)(x, y) \in L^{1}(\mathbb{R})$ for a.e. $x, y \in \mathbb{R}^{3}$. Let $F(\lambda)=$ $F_{1}(\lambda) \circ F_{2}(\lambda)$ with kernel $K(\lambda)$. Then

$$
\int_{-\infty}^{\infty}\||\widehat{K}(\xi)|\|_{2 \rightarrow 2} d \xi \leq\left[\int_{-\infty}^{\infty}\left\|\left|\widehat{K_{1}}(\xi)\right|\right\|_{2 \rightarrow 2} d \xi\right]\left[\int_{-\infty}^{\infty}\left\|\left|\widehat{K_{2}}(\xi)\right|\right\|_{2 \rightarrow 2} d \xi\right] .
$$

Proof. Without loss of generality we can assume that the right hand side is finite. Note that

$$
\left\|\int_{-\infty}^{\infty}\left|\widehat{K_{j}}(\xi)\right| d \xi\right\|_{2 \rightarrow 2} \leq \int_{-\infty}^{\infty}\left\|\left|\widehat{K}_{j}(\xi)\right|\right\|_{2 \rightarrow 2} d \xi<\infty, \quad j=1,2 .
$$

This implies that

$$
\begin{aligned}
& \int_{-\infty}^{\infty}\left|\widehat{K}_{j}(\xi)(x, y)\right| d \xi<\infty, \quad j=1,2, \\
& \int_{\mathbb{R}^{3}} \int_{\mathbb{R}^{2}}\left|\widehat{K}_{1}(\xi)\left(x, x_{1}\right)\right|\left|\widehat{K}_{2}(\eta)\left(x_{1}, y\right)\right| d \xi d \eta d x_{1}<\infty \\
& \sup _{\lambda \in \mathbb{R}} \int_{\mathbb{R}^{3}}\left|K_{1}(\lambda)\left(x, x_{1}\right) K_{2}(\lambda)\left(x_{1}, y\right)\right| d x_{1}<\infty
\end{aligned}
$$

for a.e. $x, y \in \mathbb{R}^{3}$. By definition, for a.e. $x_{1}, x_{3} \in \mathbb{R}^{3}$,

$$
K(\lambda)\left(x_{1}, x_{3}\right)=\int_{\mathbb{R}^{3}} K_{1}(\lambda)\left(x_{1}, x_{2}\right) K_{2}(\lambda)\left(x_{2}, x_{3}\right) d x_{2}
$$


and $K(\cdot)\left(x_{1}, x_{3}\right) \in L^{\infty}(\mathbb{R}) \cap L^{1}(\mathbb{R})$ by (34) and the compact support assumption in $\lambda$. Moreover, for a.e. $\xi \in \mathbb{R}$,

$$
\hat{K}(\xi)\left(x_{1}, x_{3}\right)=\int_{\mathbb{R}^{3}} \int_{-\infty}^{\infty} \hat{K}_{1}(\xi-\eta)\left(x_{1}, x_{2}\right) \hat{K}_{2}(\eta)\left(x_{2}, x_{3}\right) d \eta d x_{2} .
$$

To see this final identity, denote the right-hand side by $F\left(\xi ; x_{1}, x_{3}\right)$. Then $F\left(\cdot ; x_{1}, x_{3}\right) \in$ $L^{1}(\mathbb{R})$ for a.e. choice of $x_{1}, x_{3}$ by $(33)$, and

$$
\begin{aligned}
\int_{-\infty}^{\infty} e^{2 \pi i \xi} F\left(\xi ; x_{1}, x_{3}\right) d \xi & =\int_{-\infty}^{\infty} \int_{\mathbb{R}^{3}} \int_{-\infty}^{\infty} e^{2 \pi i(\xi-\eta)} \\
& \hat{K}_{1}(\xi-\eta)\left(x_{1}, x_{2}\right) e^{2 \pi i \eta} \hat{K}_{2}(\eta)\left(x_{2}, x_{3}\right) d \eta d x_{2} d \xi \\
& =\int_{\mathbb{R}^{3}} K_{1}(\lambda)\left(x_{1}, x_{2}\right) K_{2}(\lambda)\left(x_{2}, x_{3}\right) d x_{2} d \lambda .
\end{aligned}
$$

The final equality sign here follows by Fubini and since $\hat{K}_{j}(\cdot)(x, y) \in L^{1}(\mathbb{R})$ for a.e. choice of $x, y$ by (33). Hence, (35) holds by uniqueness of the Fourier transform. The lemma now follows by putting absolute values inside of (35) and duality.

Note that $\frac{d}{d \lambda}\left[\chi_{\lambda_{0}}(\lambda) E(\lambda)\right]$ is a sum of operators each of which is a composition of operators from the list below (here $\chi(\lambda)$ is a suitably chosen smooth cutoff supported in $\left.\left[-\lambda_{0}, \lambda_{0}\right]\right)$ :

$$
\begin{aligned}
& F_{1}(\lambda)=\chi(\lambda)\left(A(\lambda)+S_{1}\right)^{-1}, \\
& F_{2}(\lambda)=\chi(\lambda) E_{1}(\lambda), \\
& F_{3}(\lambda)=\chi(\lambda) S_{1} m(\lambda)^{-1} S_{1}, \\
& F_{4}(\lambda)=\chi(\lambda) S_{1} E_{2}(\lambda) S_{1},
\end{aligned}
$$

and their $\lambda$ derivatives. Moreover, we leave it to the reader to check that for each of the combinations that contribute to $E(\lambda)$ the hypotheses of Lemma 8 are fulfilled. Therefore, in light of Lemma 8, the following lemma completes the analysis of $K_{3}$.

Lemma 9. For each of the operators $F_{j}, j=1,2,3,4$ above,

$$
\int_{-\infty}^{\infty}\left\|\left|\widehat{F_{j}}(\xi)\right|\right\|_{2 \rightarrow 2} d \xi<\infty .
$$

The same statement is valid for their $\lambda$ derivatives, too.

Proof. We omit the analysis of $F_{1}$ and $F_{3}$. Recall that

$$
\begin{aligned}
F_{2}(\lambda) & =\chi(\lambda) E_{1}(\lambda)=\chi(\lambda) \frac{\left(A(\lambda)+S_{1}\right)^{-1}-\left(A_{0}+S_{1}\right)^{-1}}{\lambda} \\
& =\chi(\lambda) \sum_{k=1}^{\infty}(-1)^{k} \lambda^{k-1}\left(A_{0}+S_{1}\right)^{-1}\left[A_{1}(\lambda)\left(A_{0}+S_{1}\right)^{-1}\right]^{k} .
\end{aligned}
$$

Let $\chi_{1}$ be a smooth cut off function which is equal to 1 in $[-1,1]$. Note that the support of $\chi$ is contained in $[-1,1]$. We have

$$
F_{2}(\lambda)=\sum_{k=1}^{\infty}(-1)^{k} \chi(\lambda) \lambda^{k-1}\left(A_{0}+S_{1}\right)^{-1}\left[\chi_{1}(\lambda) A_{1}(\lambda)\left(A_{0}+S_{1}\right)^{-1}\right]^{k} .
$$


Using Lemma 8 and Young's inequality, we obtain

$$
\begin{aligned}
& \int_{-\infty}^{\infty}\left\|\widehat{F_{2}}(\xi) \mid\right\|_{2 \rightarrow 2} d \xi \leq \\
& \left.\sum_{k=1}^{\infty}\left\|\left(\chi \widehat{(\lambda) \lambda^{k}-1}\right)\right\|_{L^{1}}\left\|\left|\left(A_{0}+S_{1}\right)^{-1}\right|\right\|_{2 \rightarrow 2}^{k+1}\left[\int_{-\infty}^{\infty} \| \widehat{\left(\chi_{1} A_{1}\right.}\right)(\xi) \mid \|_{2 \rightarrow 2} d \xi\right]^{k} .
\end{aligned}
$$

By Remark 1, $\left|\left(A_{0}+S_{1}\right)^{-1}\right|$ is bounded on $L^{2}$. Also note that

$$
\begin{aligned}
\left\|\left(\chi \widehat{(\lambda) \lambda^{k}-1}\right)\right\|_{L^{1}} & \lesssim\left\|(1+|\xi|)\left(\chi \widehat{(\lambda) \lambda^{k}-1}\right)(\xi)\right\|_{L^{2}} \\
& \lesssim\left\|\chi(\lambda) \lambda^{k-1}\right\|_{2}+\left\|\frac{d}{d \lambda}\left(\chi(\lambda) \lambda^{k-1}\right)\right\|_{2} \\
& \lesssim \lambda_{0}^{k} .
\end{aligned}
$$

Below, we prove that

$$
\int_{-\infty}^{\infty}\left\|\left(\widehat{\chi_{1} A_{1}}\right)(\xi) \mid\right\|_{2 \rightarrow 2} d \xi \lesssim 1
$$

If $\lambda_{0}$ is chosen sufficiently small, using (37) and (38) in (36) completes the proof of the lemma for $F_{2}$. Recall that

$$
\begin{aligned}
A_{1}(\lambda)(x, y) & =v(x) \frac{e^{i \lambda|x-y|}-1}{4 \pi \lambda|x-y|} v(y) \\
& =\frac{1}{4 \pi i} v(x) v(y) \int_{0}^{1} e^{i \lambda|x-y| b} d b .
\end{aligned}
$$

Therefore,

$$
\left.\widehat{\left(\chi_{1} A_{1}\right.}\right)(\xi)(x, y)=\frac{1}{4 \pi i} v(x) v(y) \int_{0}^{1} \widehat{\chi_{1}}(\xi-|x-y| b) d b .
$$

Hence by Schur's test, we have

$$
\int_{-\infty}^{\infty}\left\|\widehat { \chi _ { 1 } A _ { 1 } } ( \xi ) \left|\left\|_{2 \rightarrow 2} d \xi \leq \int_{-\infty}^{\infty} \sup _{x} \int_{\mathbb{R}^{3}} \int_{0}^{1}\left|\widehat{\chi_{1}}(\xi-|x-y| b)\|v(x)\| v(y)\right| d b d y d \xi .\right.\right.\right.
$$

Since $\chi_{1}$ is a Schwarz function, we have (for each $N \in \mathbb{N}$ )

$$
\int_{0}^{1}\left|\widehat{\chi_{1}}(\xi-|x-y| b)\right| d b \lesssim \begin{cases}\langle x-y\rangle^{-1}, & |\xi|<|x-y| \\ \langle\xi\rangle^{-N}, & |\xi|>|x-y|\end{cases}
$$

We also have

$$
|v(x)||v(y)| \lesssim\langle x\rangle^{-\beta / 2}\langle y\rangle^{-\beta / 2} \lesssim\langle x-y\rangle^{-\beta / 2}
$$

Using this inequality and (40) in (39), we obtain

$$
\begin{aligned}
(39) & \lesssim \int_{-\infty}^{\infty} \sup _{x} \int_{\mathbb{R}^{3}}\langle x-y\rangle^{-\beta / 2}\left\{\begin{array}{cc}
\langle x-y\rangle^{-1}, & |\xi|<|x-y| \\
\langle\xi\rangle^{-N}, & |\xi|>|x-y|
\end{array}\right\} d y d \xi \\
& =\int_{-\infty}^{\infty} \int_{\mathbb{R}^{3}}\langle y\rangle^{-\beta / 2}\left\{\begin{array}{cc}
\langle y\rangle^{-1}, & |\xi|<|y| \\
\langle\xi\rangle^{-N}, & |\xi|>|y|
\end{array}\right\} d y d \xi \\
& <\infty
\end{aligned}
$$

provided $\beta>6$, i.e. $|V(x)| \lesssim\langle x\rangle^{-6-}$. To see the last inequality, fix $\xi$ and consider the integral in $y$ in the regions $\{|y|<|\xi|\}$ and $\{|y|>|\xi|\}$ separately. 
Next, we consider $F_{4}$ :

$$
F_{4}(\lambda)=\chi(\lambda) S_{1} E_{2}(\lambda) S_{1}=\chi(\lambda) S_{1} \sum_{k=1}^{\infty}(-1)^{k} \lambda^{k-1} m(0)^{-1}\left[m_{1}(\lambda) m(0)^{-1}\right]^{k} S_{1} .
$$

Arguing as in the case of $F_{2}$, it suffices to prove that

$$
\int_{-\infty}^{\infty}\left\|\left|\left(\widehat{\chi_{1} m_{1}}\right)(\xi)\right|\right\|_{2 \rightarrow 2} d \xi \lesssim 1
$$

where $\chi_{1}$ is a smooth cut off function which is equal to 1 in the support of $\chi$ (i.e. in $\left.\left[-\lambda_{0}, \lambda_{0}\right]\right)$ and which is supported in $\left[-\lambda_{1}, \lambda_{1}\right]$. Recall that

$$
m_{1}(\lambda)=S_{1} \frac{A_{1}(\lambda)-A_{1}(0)}{\lambda} S_{1}+\sum_{j=1}^{\infty} S_{1}(-1)^{j} \lambda^{j-1}\left(A_{1}(\lambda)\left(A_{0}+S_{1}\right)^{-1}\right)^{j+1} S_{1} .
$$

The second summand can be analyzed as above (here $\lambda_{1}$ is chosen sufficiently small to guarantee the convergence of the series, and than we choose $\lambda_{0}$ even smaller). Now, we consider the first summand. Note that

$$
\begin{aligned}
A_{2}(\lambda)(x, y) & :=\frac{A_{1}(\lambda)-A_{1}(0)}{\lambda}(x, y) \\
& =v(x) \frac{e^{i \lambda|x-y|}-i \lambda|x-y|-1}{\lambda^{2}|x-y|} v(y) \int_{0}^{1}(1-b) e^{i \lambda|x-y| b} d b .
\end{aligned}
$$

Therefore, using (40) and (41), we obtain

$$
\begin{aligned}
\left|\chi_{1} \widehat{S_{1} A_{2}} S_{1}(\xi)(x, y)\right| & =|v(x)| x-y\left|v(y) \int_{0}^{1}(1-b) \widehat{\chi_{1}}(\xi-b|x-y|) d b\right| \\
& \lesssim\langle x-y\rangle^{1-\beta / 2} \begin{cases}\langle x-y\rangle^{-1}, & |\xi|<|x-y| \\
\langle\xi\rangle^{-N}, & |\xi|>|x-y|\end{cases}
\end{aligned}
$$

Using this and Schur's test as before, we have

$$
\begin{aligned}
\int_{-\infty}^{\infty}\left\|\left|\chi_{1} \widehat{S_{1} A_{2}} S_{1}(\xi)\right|\right\|_{2 \rightarrow 2} d \xi & \lesssim \int_{-\infty}^{\infty} \sup _{x} \int_{\mathbb{R}^{3}}\langle x-y\rangle^{1-\beta / 2} \\
& \left\{\begin{array}{ll}
\langle x-y\rangle^{-1}, & |\xi|<|x-y| \\
\langle\xi\rangle^{-N}, & |\xi|>|x-y|
\end{array}\right\} d y<\infty
\end{aligned}
$$

provided $\beta>8$, i.e. $|V(x)| \lesssim\langle x\rangle^{-8-}$.

Next, we deal with $\frac{d}{d \lambda} F_{j}(\lambda)$. Once again we omit the analysis of $F_{1}$ and $F_{3}$. Note that

$$
\begin{aligned}
\frac{d}{d \lambda} F_{2}(\lambda)= & \sum_{k=1}^{\infty}(-1)^{k} \frac{d}{d \lambda}\left(\chi(\lambda) \lambda^{k-1}\right)\left(A_{0}+S_{1}\right)^{-1}\left[A_{1}(\lambda)\left(A_{0}+S_{1}\right)^{-1}\right]^{k} \\
+ & \sum_{k=1}^{\infty}(-1)^{k} \chi(\lambda) \lambda^{k-1}\left(A_{0}+S_{1}\right)^{-1} \times \\
& \times \sum_{j=1}^{k}\left[A_{1}(\lambda)\left(A_{0}+S_{1}\right)^{-1}\right]^{j-1}\left[\frac{d}{d \lambda} A_{1}(\lambda)\left(A_{0}+S_{1}\right)^{-1}\right]\left[A_{1}(\lambda)\left(A_{0}+S_{1}\right)^{-1}\right]^{k-j}
\end{aligned}
$$


Arguing as above, it suffices to prove that

$$
\int_{-\infty}^{\infty}\left\|\left|\left(\widehat{\chi_{1}\left(A_{1}\right)^{\prime}}\right)(\xi)\right|\right\|_{2 \rightarrow 2} d \xi \lesssim 1
$$

Note that

$$
\begin{aligned}
\frac{d}{d \lambda} A_{1}(\lambda)(x, y) & =-v(x) \frac{e^{i \lambda|x-y|}-i \lambda|x-y| e^{i \lambda|x-y|}-1}{\lambda^{2}|x-y|} v(y) \\
& =-v(x) \frac{e^{i \lambda|x-y|}-i \lambda|x-y|-1}{\lambda^{2}|x-y|} v(y)+i v(x) \frac{e^{i \lambda|x-y|}-1}{\lambda} v(y) \\
& =-A_{2}(\lambda)+i \widetilde{A}_{1}(\lambda)
\end{aligned}
$$

These are similar to the terms treated above. Therefore (44) holds provided $|V(x)| \lesssim\langle x\rangle^{-8-}$.

Finally, we analyze $\frac{d}{d \lambda} F_{4}(\lambda)$. In view of the preceding, it suffices to prove that

$$
\int_{-\infty}^{\infty}\left\|\left(\widehat{\chi_{1}\left(A_{2}\right)^{\prime}}\right)(\xi) \mid\right\|_{2 \rightarrow 2} d \xi \lesssim 1
$$

We have

$$
\begin{aligned}
\frac{d}{d \lambda} A_{2}(\lambda)(x, y)= & v(x) i \frac{e^{i \lambda|x-y|}-1}{\lambda^{2}} v(y)-2 v(x) \frac{e^{i \lambda|x-y|}-i \lambda|x-y|-1}{\lambda^{3}|x-y|} v(y) \\
= & -2 v(x) \frac{e^{i \lambda|x-y|}+\frac{1}{2} \lambda^{2}|x-y|^{2}-i \lambda|x-y|-1}{\lambda^{3}|x-y|} v(y) \\
& +i v(x) \frac{-i \lambda|x-y|+e^{i \lambda|x-y|}-1}{\lambda^{2}} v(y)
\end{aligned}
$$

These are treated as before; (45) holds provided $|V(x)| \lesssim\langle x\rangle^{-10-}$.

2.3. The general case. We now turn to the proof of Theorem 2 . In view of (5), (14), and (15), the coefficient of the $\lambda^{-2}$ power in (5) equals $R_{0}(0) v \Gamma_{1}(0) S_{1} \Gamma_{2}(0) S_{2} b(0)^{-1} S_{2} \Gamma_{2}(0) S_{1} \Gamma_{1}(0) v R_{0}(0)=-G_{0} v S_{2}\left[S_{2} v G_{2} v S_{2}\right]^{-1} S_{2} v G_{0}$.

Lemma 10. The operator $G_{0} v S_{2}\left[S_{2} v G_{2} v S_{2}\right]^{-1} S_{2} v G_{0}$ equals the orthogonal pojection in $L^{2}\left(\mathbb{R}^{3}\right)$ onto the eigenspace of $H=-\Delta+V$ at zero energy.

Proof. Let $\left\{\psi_{j}\right\}_{j=1}^{J}$ be an orthonormal basis in $\operatorname{Ran}\left(S_{2}\right)$. By Lemmas 5 and 6 ,

$$
\psi_{j}+w G_{0} v \psi_{j}=0 \quad \forall 1 \leq j \leq J
$$

and we can write $\psi_{j}=w \phi_{j}$ for $1 \leq j \leq J$ where $\phi_{2} \in L^{2}$, and

$$
\int V \phi_{j} d x=\int v \psi_{j} d x=0 .
$$

Moreover, the $\left\{\phi_{j}\right\}_{j=1}^{J}$ are linearly independent and they satisfy

$$
\phi_{j}+G_{0} V \phi_{j}=0
$$

for all $1 \leq j \leq J$. Since for any $f \in L^{2}\left(\mathbb{R}^{3}\right), S_{2} f=\sum_{j=1}^{J}\left\langle f, \psi_{j}\right\rangle \psi_{j}$, we conclude that

$$
S_{2} v G_{0} f=\sum_{j=1}^{J}\left\langle f, G_{0} v \psi_{j}\right\rangle \psi_{j}=-\sum_{j=1}^{J}\left\langle f, \phi_{j}\right\rangle \psi_{j} .
$$


Let $A=\left\{A_{i j}\right\}_{i, j=1}^{J}$ denote the matrix of the Hermitian operator

$$
S_{2} v G_{2} v S_{2}=\frac{1}{8 \pi} S_{2} v(x)|x-y| v(y) S_{2}
$$

relative to the basis $\left\{\psi_{j}\right\}_{j=1}^{J}$. Since $\int_{\mathbb{R}^{3}} v \psi_{j} d x=0$, the proof of Lemma 7 shows that

$$
\begin{aligned}
A_{i j} & =\left\langle\psi_{i}, S_{2} v G_{2} v S_{2} \psi_{j}\right\rangle=\left\langle G_{0} v \psi_{i}, G_{0} v \psi_{j}\right\rangle \\
& =\left\langle G_{0} V \phi_{i}, G_{0} V \phi_{j}\right\rangle=\left\langle\phi_{i}, \phi_{j}\right\rangle .
\end{aligned}
$$

Let

$$
Q:=G_{0} v S_{2}\left[S_{2} v G_{2} v S_{2}\right]^{-1} S_{2} v G_{0}
$$

Then for any $f \in L^{2}\left(\mathbb{R}^{3}\right)$,

$$
\begin{aligned}
Q f & =-\sum_{j=1}^{J} G_{0} v S_{2}\left[S_{2} v G_{2} v S_{2}\right]^{-1} \psi_{j}\left\langle f, \phi_{j}\right\rangle \\
& =-\sum_{i, j=1}^{J} G_{0} v S_{2} \psi_{i}\left(A^{-1}\right)_{i j}\left\langle f, \phi_{j}\right\rangle=\sum_{i, j=1}^{J} \phi_{i}\left(A^{-1}\right)_{i j}\left\langle f, \phi_{j}\right\rangle .
\end{aligned}
$$

In particular,

$$
Q \phi_{k}=\sum_{i, j=1}^{J} \phi_{i}\left(A^{-1}\right)_{i j}\left\langle\phi_{k}, \phi_{j}\right\rangle=\sum_{i, j=1}^{J} \phi_{i}\left(A^{-1}\right)_{i j} A_{j k}=\phi_{k}
$$

for all $1 \leq k \leq J$. The conclusion is that $\operatorname{Ran} Q=\operatorname{span}\left\{\phi_{j}\right\}_{j=1}^{J}$, and that $Q=\operatorname{Id}$ on $\operatorname{Ran} Q$. Since $Q$ is Hermitian, it is the orthogonal projection onto $\operatorname{span}\left\{\phi_{j}\right\}_{j=1}^{J}$, as claimed.

This has the following simple and standard consequence for the spectral measure.

Corollary 11. Let $-\infty<\lambda_{N}<\lambda_{N-1}<\ldots<\lambda_{1}<\lambda_{0} \leq 0$ be the finitely many eigenvalues of $H=-\Delta+V$. Let $P_{\lambda_{j}}$ denote the orthogonal projection in $L^{2}\left(\mathbb{R}^{3}\right)$ onto the eigenspace of $H$ corresponding to the eigenvalue $\lambda_{j}$. Then

$$
e^{i t H}=\sum_{j=0}^{N} e^{i t \lambda_{j}} P_{\lambda_{j}}+\frac{1}{2 \pi i} \int_{0}^{\infty} e^{i t \lambda}\left[R_{V}^{+}(\lambda)-R_{V}^{-}(\lambda)\right] d \lambda .
$$

Moreover,

$$
R_{V}^{+}(\lambda)-R_{V}^{-}(\lambda)=O_{L^{2}}\left(\lambda^{-\frac{1}{2}}\right)
$$

as $\lambda \rightarrow 0+$ so that the integral in (46) is absolutely convergent at $\lambda=0$.

PROOF. Start from the expression

$$
e^{i t H}=\frac{1}{2 \pi i} \int_{0}^{\infty} e^{i t \lambda}\left[R_{V}(\lambda+i \epsilon)-R_{V}(\lambda-i \epsilon)\right] d \lambda,
$$

which is valid for all $\epsilon>0$ (via the spectral theorem, for example). The formula (46) follows by passing to the limit $\epsilon \rightarrow 0$. Indeed, the projections arise as Cauchy integrals

$$
P_{\lambda_{j}} \frac{1}{2 \pi i} \oint_{\gamma_{j}} \frac{d z}{z-\lambda_{j}}=P_{\lambda_{j}}
$$


where $\gamma_{j}$ is a small circle surrounding $\lambda_{j}$. We need to invoke Lemma 10 in case $\lambda_{0}=0$, since it determines the coefficient of the $z^{-1}$ singularity in the asymptotic expansion of the resolvent. Once we subtract that singularity, what remains is $O\left(|z|^{-\frac{1}{2}}\right)$, as claimed.

The point of Lemma 10 and Corollary 11 is really to prove (47), since (46) is of course obvious. One can also deduce Lemma 10 from the proof of the corollary starting from (46), since the most singular power $z^{-1}$ must lead to the projection onto the eigenspace. However, we have chosen to give these direct proofs.

Proof of TheOrem 2. In view of (15),

$$
\begin{aligned}
A(\lambda)^{-1} & =\Gamma_{1}(\lambda) \\
& +\frac{1}{\lambda} \Gamma_{1}(\lambda) S_{1} \Gamma_{2}(\lambda) S_{1} \Gamma_{1}(\lambda) \\
& +\frac{1}{\lambda^{2}}\left[\Gamma_{1}(\lambda) S_{1} \Gamma_{2}(\lambda) S_{2} b(\lambda)^{-1} S_{2} \Gamma_{2}(\lambda) S_{1} \Gamma_{1}(\lambda)-S_{2} b(0)^{-1} S_{2}\right] \\
& +\frac{1}{\lambda^{2}} S_{2} b(0)^{-1} S_{2} .
\end{aligned}
$$

Inserting this into (5) leads to

$R_{V}\left(\lambda^{2}\right)=R_{0}\left(\lambda^{2}\right)-R_{0}\left(\lambda^{2}\right) v \Gamma_{1}(\lambda) v R_{0}\left(\lambda^{2}\right)$

$$
\begin{aligned}
& -\frac{1}{\lambda} R_{0}\left(\lambda^{2}\right) v \Gamma_{1}(\lambda) S_{1} \Gamma_{2}(\lambda) S_{1} \Gamma_{1}(\lambda) v R_{0}\left(\lambda^{2}\right) \\
& -\frac{1}{\lambda^{2}} R_{0}\left(\lambda^{2}\right) v\left[\Gamma_{1}(\lambda) S_{1} \Gamma_{2}(\lambda) S_{2} b(\lambda)^{-1} S_{2} \Gamma_{2}(\lambda) S_{1} \Gamma_{1}(\lambda)-S_{2} b(0)^{-1} S_{2}\right] v R_{0}\left(\lambda^{2}\right) \\
& -\frac{1}{\lambda^{2}}\left(R_{0}\left(\lambda^{2}\right)-G_{0}\right) v S_{2} b(0)^{-1} S_{2} v R_{0}\left(\lambda^{2}\right)-\frac{1}{\lambda^{2}} G_{0} v S_{2} b(0)^{-1} S_{2} v\left(R_{0}\left(\lambda^{2}\right)-G_{0}\right) \\
& -\frac{1}{\lambda^{2}} P_{0} .
\end{aligned}
$$

The three terms up to and including (48) have already been covered in Subsection 2.2. Indeed, the only difference here is that we need to incorporate $S_{2}$ into the expression (21):

$$
\begin{aligned}
m(\lambda)^{-1} & =\left(m(0)+S_{2}\right)^{-1}+\sum_{k=1}^{\infty}(-1)^{k} \lambda^{k}\left(m(0)+S_{2}\right)^{-1}\left[m_{1}(\lambda)\left(m(0)+S_{2}\right)^{-1}\right]^{k} \\
& =:\left(m(0)+S_{2}\right)^{-1}+\lambda E_{2}(\lambda) .
\end{aligned}
$$

The term (51) has been dealt with in Corollary 11. Now, we consider (50). Note that when we plug $R_{V}$ into (3), then the term corresponding to the first summand in 
(50) is (with the notation $S=S_{2} b(0)^{-1} S_{2}, a_{1}=\left|y-y_{1}\right|$ and $a_{2}=\left|x-x_{1}\right|+\left|y-y_{1}\right|$ )

$$
\begin{aligned}
& \frac{-1}{\pi i} \int_{-\infty}^{\infty} \int_{\mathbb{R}^{6}} e^{i t \lambda^{2}} \chi_{\lambda_{0}}(\lambda) \frac{e^{i \lambda\left|x-x_{1}\right|}-1}{\lambda 4 \pi\left|x-x_{1}\right|} \frac{e^{i \lambda\left|y-y_{1}\right|}}{4 \pi\left|y-y_{1}\right|} v\left(x_{1}\right) S\left(x_{1}, y_{1}\right) v\left(y_{1}\right) d x_{1} d y_{1} d \lambda \\
& =\frac{-1}{16 \pi^{3}} \int_{-\infty}^{\infty} \int_{\mathbb{R}^{6}} e^{i t \lambda^{2}} \chi_{\lambda_{0}}(\lambda) \frac{\sin \left(\lambda a_{2}\right)-\sin \left(\lambda a_{1}\right)}{\lambda\left|x-x_{1}\right|\left|y-y_{1}\right|} v\left(x_{1}\right) S\left(x_{1}, y_{1}\right) v\left(y_{1}\right) d x_{1} d y_{1} d \lambda \\
& =\frac{-1}{16 \pi^{3}} \int_{-\infty}^{\infty} \int_{\mathbb{R}^{6}} e^{i t \lambda^{2}} \chi_{\lambda_{0}}(\lambda) \int_{a_{1}}^{a_{2}} \cos (\lambda b) d b \frac{v\left(x_{1}\right) S\left(x_{1}, y_{1}\right) v\left(y_{1}\right)}{\left|x-x_{1}\right|\left|y-y_{1}\right|} d x_{1} d y_{1} d \lambda \\
& =: t^{-1 / 2} F_{1, t}(x, y) .
\end{aligned}
$$

Arguing as in (25), we obtain

$$
\begin{aligned}
& \left|F_{1, t}(x, y)\right| \\
& =c\left|\int_{-\infty}^{\infty} \int_{\mathbb{R}^{6}} e^{i u^{2} / 4 t} \int_{a_{1}}^{a_{2}}\left[\widehat{\chi_{\lambda_{0}}}(u+b)+\widehat{\chi \lambda_{0}}(u-b)\right] d b \frac{v\left(x_{1}\right) S\left(x_{1}, y_{1}\right) v\left(y_{1}\right)}{\left|x-x_{1}\right|\left|y-y_{1}\right|} d x_{1} d y_{1} d u\right| \\
& \lesssim \int_{\mathbb{R}^{6}} \int_{a_{1}}^{a_{2}} \int_{-\infty}^{\infty}\left|\widehat{\chi_{\lambda_{0}}}(u+b)+\widehat{\chi \lambda_{0}}(u-b)\right| \frac{\left|v\left(x_{1}\right)\right|\left|S\left(x_{1}, y_{1}\right)\right|\left|v\left(y_{1}\right)\right|}{\left|x-x_{1}\right|\left|y-y_{1}\right|} d u d b d x_{1} d y_{1} \\
& \lesssim\left\|\widehat{\chi \lambda_{0}}\right\|_{1} \int_{\mathbb{R}^{6}} \frac{\left|v\left(x_{1}\right)\right|\left|S\left(x_{1}, y_{1}\right)\right|\left|v\left(y_{1}\right)\right|}{\left|y-y_{1}\right|} d x_{1} d y_{1} \\
& \lesssim 1
\end{aligned}
$$

This inequality holds independently of $t, x$ and $y$. Therefore,

$$
\sup _{t}\left\|F_{1, t}\right\|_{L^{1} \rightarrow L^{\infty}} \lesssim 1 \text { and } \lim _{t \rightarrow \infty} F_{1, t}(x, y)=c \int_{\mathbb{R}^{6}} \frac{v\left(x_{1}\right) S\left(x_{1}, y_{1}\right) v\left(y_{1}\right)}{\left|y-y_{1}\right|} d x_{1} d y_{1} .
$$

The second summand in (50) can be treated similarly.

Now, we consider (49); it can be written as

$$
\text { (49) }=\lambda^{-1} R_{0}\left(\lambda^{2}\right) v E_{3}(\lambda) v R_{0}\left(\lambda^{2}\right)
$$

with

$$
\lambda E_{3}(\lambda):=-\Gamma_{1}(\lambda) S_{1} \Gamma_{2}(\lambda) S_{2} b(\lambda)^{-1} S_{2} \Gamma_{2}(\lambda) S_{1} \Gamma_{1}(\lambda)+S_{2} b(0)^{-1} S_{2} .
$$

Clearly, the terms resulting from $E_{3}$ resemble $K_{3}$ from (23). However, we do not have an extra $\lambda$ at our disposal, which implies that instead of (29) we will only obtain a $t^{-\frac{1}{2}}$ power. The details are as follows: if we plug $R_{V}$ into (3), then the term corresponding to (49) is (up to constants)

$$
\int_{-\infty}^{\infty} \int_{\mathbb{R}^{6}} e^{i t \lambda^{2}} \chi_{\lambda_{0}}(\lambda) \frac{e^{i \lambda\left|x-x_{1}\right|}}{\left|x-x_{1}\right|} \frac{e^{i \lambda\left|y-y_{1}\right|}}{\left|y-y_{1}\right|} v\left(x_{1}\right) E_{3}(\lambda)\left(x_{1}, y_{1}\right) v\left(y_{1}\right) d x_{1} d y_{1} d \lambda .
$$

By the arguments that lead from (30) to (32), we conclude that the absolute value of this expression does not exceed

$$
|t|^{-\frac{1}{2}} \int_{-\infty}^{\infty}\left\|\left|\widehat{\chi_{\lambda_{0}} E_{3}}(\xi)\right|\right\|_{L^{2} \rightarrow L^{2}} d \xi
$$


uniformly in $x, y \in \mathbb{R}^{3}$. To bound this integral, we use Lemma 8. Write

$$
\begin{aligned}
E_{3}(\lambda) & =-\lambda^{-1}\left(\Gamma_{1}(\lambda)-\Gamma_{1}(0)\right) S_{1} \Gamma_{2}(\lambda) S_{2} b(\lambda)^{-1} S_{2} \Gamma_{2}(\lambda) S_{1} \Gamma_{1}(\lambda) \\
& -S_{1} \lambda^{-1}\left(\Gamma_{2}(\lambda)-\Gamma_{2}(0)\right) S_{2} b(\lambda)^{-1} S_{2} \Gamma_{2}(\lambda) S_{1} \Gamma_{1}(\lambda) \\
& -S_{2} \lambda^{-1}\left(b(\lambda)^{-1}-b(0)^{-1}\right) S_{2} \Gamma_{2}(\lambda) S_{1} \Gamma_{1}(\lambda) \\
& -S_{2} b(0)^{-1} S_{2} \lambda^{-1}\left(\Gamma_{2}(\lambda)-\Gamma_{2}(0)\right) S_{1} \Gamma_{1}(\lambda) \\
& -S_{2} b(0)^{-1} S_{2} \lambda^{-1}\left(\Gamma_{1}(\lambda)-\Gamma_{1}(0)\right) .
\end{aligned}
$$

Consequently, we need to prove the bound of Lemma 9 for the following basic building blocks (we dropped the subscript $\lambda_{0}$ ):

$$
\begin{aligned}
F_{1}(\lambda) & =\chi(\lambda) \Gamma_{1}(\lambda)=\chi(\lambda)\left(A(\lambda)+S_{1}\right)^{-1} \\
F_{2}(\lambda) & =\chi(\lambda) \lambda^{-1}\left(\Gamma_{1}(\lambda)-\Gamma_{1}(0)\right)=\chi(\lambda) \lambda^{-1}\left(\left(A(\lambda)+S_{1}\right)^{-1}-\left(A_{0}+S_{1}\right)^{-1}\right) \\
F_{3}(\lambda) & =\chi(\lambda) S_{1} \Gamma_{2}(\lambda) S_{1}=\chi(\lambda) S_{1}\left(m(\lambda)+S_{2}\right)^{-1} S_{1} \\
F_{4}(\lambda) & =\chi(\lambda) \lambda^{-1} S_{1}\left(\Gamma_{2}(\lambda)-\Gamma_{2}(0)\right) S_{1}=\chi(\lambda) \lambda^{-1} S_{1}\left(\left(m(\lambda)+S_{2}\right)^{-1}\right. \\
& \left.-\left(m(0)+S_{2}\right)^{-1}\right) S_{1}
\end{aligned}
$$

as well as

$$
\begin{aligned}
& F_{5}(\lambda)=\chi(\lambda) S_{2} b(\lambda)^{-1} S_{2}=\chi(\lambda) S_{2}\left(b(0)+\lambda b_{1}(\lambda)\right)^{-1} S_{2} \\
& F_{6}(\lambda)=\chi(\lambda) S_{2} \lambda^{-1}\left(b(\lambda)^{-1}-b(0)^{-1}\right) S_{2} .
\end{aligned}
$$

The functions $F_{j}$ with $1 \leq j \leq 4$ were already discussed in Lemma 9 . The only difference here is the appearance of $S_{2}$ in $F_{3}$ and $F_{4}$ (for the function $E_{2}$ see (21)). But this does not effect the bounds from Lemma 9 , which implies that we only need to prove the following claims concerning the new terms $F_{5}$ and $F_{6}$ :

$$
\max _{j=5,6} \int_{-\infty}^{\infty}\left\|\left|\widehat{F_{j}}(\xi)\right|\right\|_{2 \rightarrow 2} d \xi<\infty .
$$

Recall that, see (13),

$$
\begin{aligned}
& b(0)=-S_{2} v G_{2} v S_{2} \\
& b(\lambda)=b(0)+\lambda b_{1}(\lambda)=b(0)\left(1+\lambda b(0)^{-1} b_{1}(\lambda)\right)
\end{aligned}
$$

$$
b_{1}(\lambda)=\frac{S_{2}\left[m_{1}(\lambda)-m_{1}(0)\right] S_{2}}{\lambda}+\frac{1}{\lambda} \sum_{k=1}^{\infty}(-1)^{k} \lambda^{k} S_{2}\left(m_{1}(\lambda)\left(m(0)+S_{2}\right)^{-1}\right)^{k+1} S_{2}
$$

$$
b(\lambda)^{-1}=\sum_{j=0}^{\infty}(-1)^{j} \lambda^{j}\left(b(0)^{-1} b_{1}(\lambda)\right)^{j} b(0)^{-1} .
$$

Applying Lemma 8 to the Neuman series in (54) shows that in order to obtain (52), we need to prove that

$$
\int_{-\infty}^{\infty}\left\|\left|\widehat{\chi_{1} b_{1}}(\xi)\right|\right\|_{2 \rightarrow 2} d \xi<\infty
$$


Another application of Lemma 8, this time to the Neuman series (53), reduces matters to proving

$$
\int_{-\infty}^{\infty}\left\|\left|\widehat{\chi_{2} m_{1}}(\xi)\right|\right\|_{2 \rightarrow 2} d \xi<\infty
$$

which was already done in (42). In both these cases, the cut-off functions $\chi_{1}, \chi_{2}$ need to be taken with sufficiently small supports. This leaves the term

$$
\frac{S_{2}\left[m_{1}(\lambda)-m_{1}(0)\right] S_{2}}{\lambda}
$$

from (53) to be considered. In view of (10) and (43),

$$
\begin{aligned}
& S_{2} \frac{m_{1}(\lambda)-m_{1}(0)}{\lambda} S_{2} \\
& =S_{2} \frac{A_{2}(\lambda)-A_{2}(0)}{\lambda} S_{2}+\sum_{k=1}^{\infty}(-1)^{k} \lambda^{k-1} S_{2}\left(A_{1}(\lambda)\left(A_{0}+S_{1}\right)^{-1}\right)^{k+1} S_{2} .
\end{aligned}
$$

By (38), and Lemma 8, the Neuman series makes a summable contribution to (52). On the other hand, the contribution of

$$
S_{2} \frac{A_{2}(\lambda)-A_{2}(0)}{\lambda} S_{2}
$$

to (52) is controlled by the bound (45), and we are done.

\section{References}

[Agm] Agmon, S. Spectral properties of Schrödinger operators and scattering theory. Ann. Scuola Norm. Sup. Pisa Cl. Sci. (4) 2 (1975), no. 2, 151-218.

[Cuc] Cuccagna, S. Stabilization of solutions to nonlinear Schrödinger equations. Comm. Pure Appl. Math. 54 (2001), no. 9, 1110-1145.

[Gol] Goldberg, M. Dispersive bounds for the three-dimensional Schrödinger equation with almost critical potentials. preprint, 2004.

[GolSch] Goldberg, M., Schlag, W. Dispersive estimates for Schrödinger operators in dimensions one and three. Comm. Math. Phys. 251, no. 1 (2004), 157 - 178.

[JenKat] Jensen, A., Kato, T. Spectral properties of Schrödinger operators and time-decay of the wave functions. Duke Math. J. 46 (1979), no. 3, 583-611.

[JenNen] Jensen, A., Nenciu, G. A unified approach to resolvent expansions at thresholds. Rev. Math. Phys. 13 (2001), no.6, 717-754.

[JouSofSog] Journé, J.-L., Soffer, A., Sogge, C. D. Decay estimates for Schrödinger operators. Comm. Pure Appl. Math. 44 (1991), no. 5, 573-604.

[Mur] Murata, M. Asymptotic expansions in time for solutions of Schrödinger-type equations J. Funct. Anal. 49 (1) (1982), 10-56.

[Rau] Rauch, J. Local decay of scattering solutions to Schrödinger's equation. Comm. Math. Phys. 61 (1978), no. 2, 149-168.

[RodSch] Rodnianski, I., Schlag, W. Time decay for solutions of Schrödinger equations with rough and time-dependent potentials. Invent. Math. 155 (2004), no. 3, 451-513.

[Sch] Schlag, W. Stable manifolds for an orbitally unstable NLS, preprint 2004.

[Yaj1] Yajima, K. The $W^{k, p}$-continuity of wave operators for Schrödinger operators. J. Math. Soc. Japan 47 (1995), no. 3, 551-581.

Department of Mathematics, University of Illinois, Urbana, IL 61801, U.S.A.

E-mail address: berdogan@math.uiuc.edu

Department of Mathematics, Caltech, Pasadena, CA 91125, U.S.A.

E-mail address: schlag@caltech.edu 